\title{
A surprising diagnosis of pancreatitis with pseudocyst associated with sudden massive effusion
}

\author{
EMINE ARGÜDER, DEMET KARNAK and OYA KAYACAN \\ Department of Chest Diseases, Ankara University School of Medicine, Ankara, Turkey
}

Received March 1, 2011; Accepted March 30, 2011

DOI: $10.3892 /$ etm.2011.248

\begin{abstract}
This article describes the case of a 40 year-old man presenting with pleuritic pain on the right side, cough, sputum and sweating symptoms. He had smoked 40 pack-years of cigarettes and consumed $140 \mathrm{ml}$ alcohol/day for 20 years. Breath sounds were diminished at the right lung base. Chest $\mathrm{X}$-ray showed right hemi-diaphragm elevation and heterogeneous opacity on the right inferior zone. Antibiotherapy was commenced for possible diagnosis of pleuropnemonia. The patient developed dyspnea after a few days. Right hemithorax filled with a parabolic shadow was observed on the chest $\mathrm{X}$-ray. Serosanguineous exudate was sampled. Very high levels of amylase and lipase levels were detected in the pleural fluid. Furthermore, magnetic resonance imaging revealed pancreatic pseudocyst near the left diaphragmatic crus. Four thousand milliliters of pleural fluid was drained using an intercostal drain within a 4-day period, and chest symptoms were relieved. Upon follow-up, suitable therapy for chronic pancreatitis was administered, and the patient was stable without any recurrence.
\end{abstract}

\section{Introduction}

Unilateral massive exudate is usually related with a malignant or infectious (parapneumonic or tuberculosis) origin. Subdiaphragmatic diseases, such as hepatic or subdiaphragmatic abscess, acute and chronic pancreatitis, are rarely noted in clinical practice, as shown in Table I $(1,2)$. Herein, we present a patient with symptoms leading to a misdiagnosis of pleuropneumonia.

\section{Case report}

A 40 year-old man presented with chest and right shoulder pain, cough, sputum and sweating symptoms, for a period of

Correspondence to: Dr Emine Argüder, Department of Chest Diseases, Ankara University School of Medicine, Yenibatı Mah. 5. Cad. 294. Sok. Diriliş Sitesi 6/7, Batikent, Ankara, Turkey

E-mail: drgullu2000@gmail.com

Key words: massive, pleural, effusion, pancreatitis, pseudocyst
1 month. Two weeks earlier, the patient had been examined in the emergency room due to epigastric pain and abdominal distension. He had smoked 40 pack-years of cigarettes and consumed $140 \mathrm{ml}$ alcohol/day for 20 years. The patient complained of delirium-like symptoms due to alcohol withdrawal. His vital signs were normal. Expansion of the right hemi-thorax was delayed. Percussion tone was dull and breath sounds were diminished at the right lung base.

Laboratory analysis showed a high level of hemoglobin $(18.2 \mathrm{~g} / \mathrm{dl})$, hematocrit $(54.2 \%)$ and white blood cells $\left(12.1 \times 10^{3} /\right.$ $\mu \mathrm{l})$. Glucose $(190 \mathrm{mg} / \mathrm{dl})$, total bilirubin $(2.34 \mathrm{mg} / \mathrm{dl})$, direct bilirubin $(0.3 \mathrm{mg} / \mathrm{dl})$ and lactate dehydrogenase $(691 \mathrm{U} / \mathrm{l})$ levels were also found to be high. Platelet count $\left(237 \times 10^{3} / \mu 1\right)$, erythrocyte sedimentation rate $(14 \mathrm{~mm} / \mathrm{h})$ and the remaining routine biochemical parameters were normal. Pulmonary function tests revealed a restrictive defect. On the chest X-ray the right hemi-diaphragm was elevated and the right costophrenic sinus was obliterated. The right hilus was widened and heterogeneous opacity was observed on the right inferior zone.

Pleuropneumonia most probably due to the aspiration was first considered as a possible diagnosis. Alcohol consumption was restricted, and antibiotics (cefuroxime axetyl $500 \mathrm{mg}$, p.o., BID) were administered. Diazepam and vitamin B6 were also supplemented in order to control any potential alcohol withdrawal symptoms. A diabetic diet was recommended to sustain the blood glucose level within an acceptable range. A few days later the patient developed severe dyspnea. Common dullness appeared on the right hemi-thorax. Right parabolic opacity was detected on the chest X-ray (Fig. 1). Computed tomography (CT) of the thorax demonstrated massive pleural effusion on the right hemi-thorax. Thoracentesis and biochemical analysis of the pleural fluid were performed (Table II). Serosanguineous exudate was sampled.

High levels of serum amylase and lipase, and a high pleura to serum ratio of these enzymes were detected. The urine amylase level was also very high. Adenosine deaminase (ADA) levels of serum and pleural fluid were unremarkable ruling out tuberculosis. Cytopathological examination of the fluid revealed mesothelial reaction. Pleural biopsy was reported as nonspecific pleuritis. On abdominal CT, a right paragastric lesion was detected. Endoscopic retrograde cholangiopancreatography was non-diagnostic. Magnetic resonance imaging (MRI) of the pancreas revealed a pseudocyst close to the left diaphragmatic crus (Fig. 2). 
Table I. Causes of unilateral, massive and exudative pleural effusions.

\begin{tabular}{lll}
\hline Common causes & \multicolumn{1}{c}{ Less common causes } & \multicolumn{1}{c}{ Rare causes } \\
\hline Malignancy & Pulmonary embolism & Yellow-nail syndrome (and other lymphatic \\
Parapneumonic effusions & Rheumatoid arthritis and other autoimmune pleuritis & disorders, lymphangioleiomyomatosis) \\
Tuberculosis & Benign asbestos effusion & Drugs \\
& Pancreatitis & Fungal infections \\
& Post-myocardial infarction & \\
& Post-coronary artery bypass graft & \\
\hline
\end{tabular}

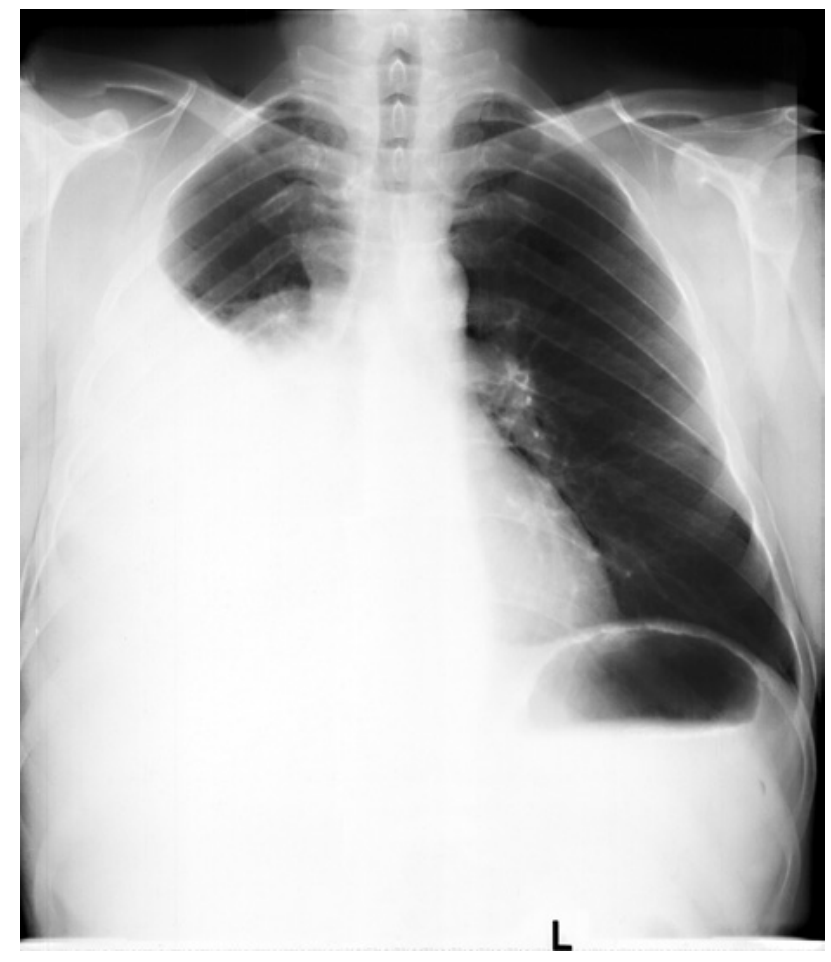

Figure 1. Chest X-ray showing homogenous parabolic shadow on the right lower zone.

Table II. Patient biochemical results of the pleural fluid analysis

\begin{tabular}{lcccc}
\hline Variable & $\begin{array}{c}\text { Serum } \\
(\mathrm{U} / \mathrm{l})\end{array}$ & $\begin{array}{c}\text { Pleural } \\
\text { fluid (U/1) }\end{array}$ & $\begin{array}{c}\text { Urine } \\
(\mathrm{U} / \mathrm{l})\end{array}$ & $\begin{array}{c}\text { Pleura/serum } \\
\text { ratio }\end{array}$ \\
\hline Amylase & 369.0 & 44,900 & 4,751 & $>1$ \\
Lipase & 465.0 & 173,000 & - & $>1$ \\
ADA & 8.3 & 11 & - & $>1$ \\
\hline
\end{tabular}

ADA, adenosine deaminase.

Four thousand milliliters of pleural fluid was drained through an intercostal drain (Pleurocane ${ }^{\circledR}$ ) during a 4-day interval, and chest symptoms were relieved. By antibiotherapy and intravenous feeding, clinical and radiological improve-

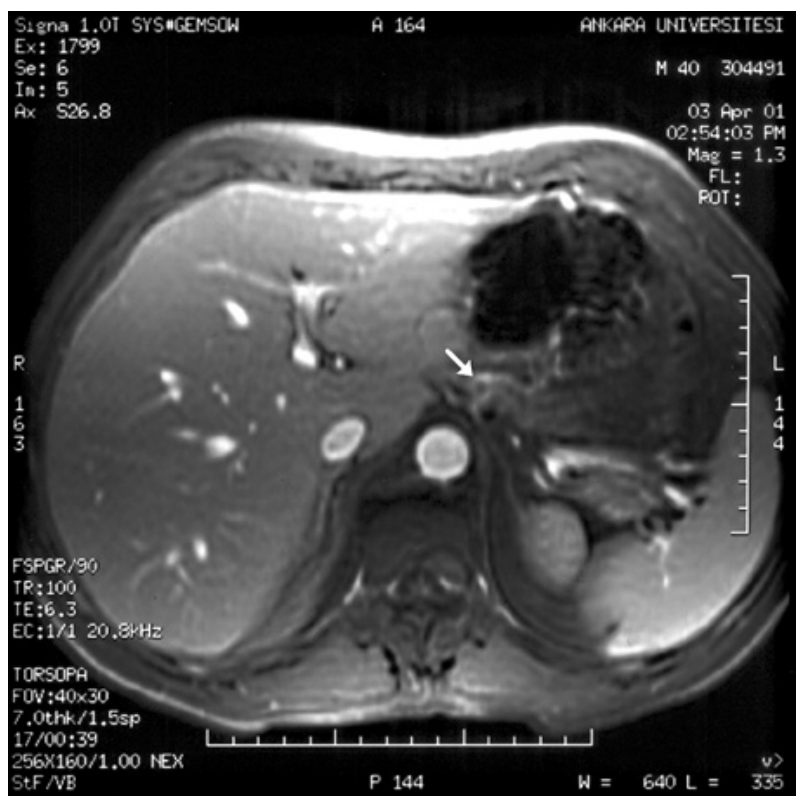

Figure 2. Pseudocyst near the left diaphragmatic crus was noted on the MRI of the pancreas.

ment was achieved. Upon follow-up, the patient was in good health, and his physical examination, laboratory and radiological findings progressively normalized.

\section{Discussion}

Pleural effusion associated with pancreatitis is usually symptomatic and inflammatory in nature. It is noted in approximately 3-17\% of cases of pancreatitis. Pleural effusion is uncommon in chronic pancreatitis $(<1 \%)$ and occurs as a consequence of a fistula or pseudocyst (3). Chronic pancreatitis occurs most often in patients with alcoholism (70-80\% of all cases). Ethanol is implicated in the secretion of insoluble pancreatic proteins that calcify and occlude the pancreatic duct $(2,4)$. Massive pleural effusion related to pancreatic pseudocyst is very rare and the incidence remains unknown (5). Recurrent pleural effusions may develop. Although effusions are generally on the left, they may also be observed on the right or bilateral side $(3,5-8)$. In chronic pancreatitis, the cause of pleural effusion is attibuted to the direct extension of a pseudocyst across the diaphragm. or by the formation of a fistulous tract between the pancreas and pleural spaces 
(9). Although a pleuropancreatic fistula was not documented, pleural effusion may have been the complication of this pseudocyst in this case.

Alcoholic pancreatitis is generally the most common cause of massive pleural fluid. There are several explanations for the pathogenesis of pancreatic duct disruption. Alcohol ingestion induces focal acute inflammation on a single branch of the pancreatic duct system and elicits the protein plug formation. If transient obstruction occurs with protein plugs, pleural collection can be observed due to leakage of the pancreatic fluid. From the retroperitoneal space, it usually moves upward due to the transdiaphragmatic pressure gradient between the abdominal and pleural cavities (10).

Pancreatic pseudocysts are localized collections of pancreatic fluid resulting from disruption of the duct or acinus. Approximately $25 \%$ of patients with chronic pancreatitis develop a pseudocyst. Patients should be administered intravenous or jejunal enteral feeding to rest the bowel and minimize pancreatic stimulation, somatostatin infusion and repeated aspiration. The cyst resolves in $70 \%$ of cases after 2 or 3 weeks. Persistent leaks to the abdominal cavity require endoscopic stenting of the pancreatic duct or surgery to drain the site of leakage if it is proximal or resection if distal $(11,12)$. In cases with pleuropancreatic fistula, chronic massive and/ or recurrent pleural effusions may develop $(9,12)$. Sometimes, chronic pancreatitis may present only with pleural effusions (5). In such cases, CT is recommended to show pancreatic parenchymal atrophy, in addition to dilatation of the pancreatic ducts, calcifications and pseudocysts (3). The pleural fluid due to chronic pancreatitis is usually bloody and contains a high level of amylase, which is predominantly a pancreatic type of isoenzymes (10). Adenocarcinoma of the lung and female genital tract, other solid neoplasms and esophageal perforation or rupture can also be the reason for high amylase levels in the fluid (9). The pancreatic pseudocyst of the present case could be detected by pancreatic MRI. Magnetic resonance cholangiopancreatography, ultrasonography and ERCP are also advocated to scan pleuropancreatic fistula $(3,13-15)$.

In chronic pancreatitis, medical treatment is mainly based on alcohol withdrawal, analgesics and restoration of normal nutritional status. Pain can be decreased, but sometimes endoscopic, radiologic or surgical procedures are required. Surgery is performed in a small group of patients when other therapeutic approaches fail. Insulin is often given for diabetes, while exocrine insufficiency is substituted by gastroresistant microgranule pancreatic extracts $(16,17)$.

Briefly, in the present case, ADA levels in serum or pleural fluid and microscopic examination for acid fast bacilli or other bacterias ruled out tuberculosis and empyema. There was no sign reflecting malignancy on thorax or abdominal CT and MRI. The patient denied any trauma or accident during the last 2 weeks. Radiological findings were prominent for pancreatic pseudocyst formation. High amylase and lipase levels detected in serum and pleural fluid indicated pancreatic inflammation. In conclusion, chronic pancreatitis, including a pseudocyst, should be considered in patients developing massive pleural effusion in case of alcohol abuse. Although it is extremely less common, chronic pancreatitis including pseudocyst should be included in the list of differential diagnosis for massive pleural effusion.

\section{References}

1. Hooper C, Lee YC, Maskell N; BTS Pleural Guideline Group: Investigation of a unilateral pleural effusion in adults: British Thoracic Society Pleural Disease Guideline 2010. Thorax 65 (Suppl 2): ii4-ii17, 2010.

2. Shan SA: The pleura. Am Rev Respir Dis 138: 184-234, 1988.

3. Materne R, Vranckx P, Pauls C, Coche EE, Deprez P and van Beers BE: Pancreaticopleural fistula: diagnosis with magnetic resonance pancreatography. Chest 117: 912-914, 2000.

4. Tierney LM Jr, McPhee SJ and Papadakis MA (eds): Current Medical Diagnosis and Treatment 2003. 42nd edition. Lange Medical Books/McGraw-Hill, p669, 2003.

5. Molinuevo JL, Moitinho E, Font MC, et al: Massive pleural effusion secondary to pancreatic-pleural fistula as first manifestation of chronic pancreatitis. Report of three cases. Med Clin 109: 222-224, 1997.

6. Kiewiet JJ, Moret M, Blok WL, Gerhards MF and de Wit LT: Two patients with chronic pancreatitis complicated by a pancreaticopleural fistula. Case Rep Gastroenterol 3: 36-42, 2009.

7. Vyas S, Gogoi D, Sinha SK, Singh P, Yadav TD and Khandelwal N: Pancreaticopleural fistula: an unusual complication of pancreatitis diagnosed with magnetic resonance cholangiopancreatography. JOP 10: 671-673, 2009.

8. Reechaipichitkul W, Bowornkitiwong T and Utchariyaprasit E: Chronic pancreatitis presenting with right pleural effusion: a case report. J Med Assoc Thai 93: 378-382, 2010.

9. Iglesias JI, Cobb J, Levey J and Rosiello RA: Recurrent left pleural effusion in a 44-year-old woman with a history of alcohol abuse. Chest 110: 547-549, 1996.

10. Akahane T, Kuriyama S, Matsumoto M, et al: Pancreatic pleural effusion with a pancreaticopleural fistula diagnosed by magnetic resonance cholangiopancreatograpy and cured by somatostatin analogue treatment. Abdom Imaging 28: 92-95, 2003.

11. Bornman $\mathrm{C}$ and Beckingham IJ: ABC of diseases of liver, pancreas, and biliary system. Chronic pancreatitis. BMJ 322: 660-663, 2001.

12. Bhasin DK, Rana SS, Chandail VS, Nanda M, Sinha SK and Nagi B: Successful resolution of a mediastinal pseudocyst and pancreatic pleural effusion by endoscopic nasopancreatic drainage. JOP 6: 359-364, 2005.

13. Siwezynski H: Chronic pleural effusion from the pancreas. Wiad Lek 51: 190-195, 1998.

14. Kumar A, Upreti L, Bhargava SK and Gupta S: Sonographic demonstration of a pancreaticopleural fistula. J Clin Ultrasound 30: 503-505, 2002.

15. Safadi BY and Marks JM: Pancreatic-pleural fistula: the role of ERCP in diagnosis and treatment. Gastrointest Endosc 51: 213-215, 2000.

16. Buscail L: Diagnosis and management of chronic pancreatitis. Rev Prat 52: 1561-1566, 2002.

17. Olakowski M, Mieczkowska-Palacz H, Olakowska E and Lampe P: Surgical management of pancreaticopleural fistulas. Acta Chir Belg 109: 735-740, 2009. 